\title{
Pressure Drop Characteristics on Gas/Liquid Two-Phase Flow in A Curved Mini-Channel Affected by The Wall Wettability*
}

\author{
Toru SUKAWA ${ }^{* *}$, Tomoya HASEGAWA ${ }^{* *}$, Kenji YOSHIDA ${ }^{* *}$ \\ and Isao KATAOKA ${ }^{* *}$ \\ ** Department of Mechanical Engineering \\ Osaka University \\ 2-1, Yamada-Oka, Suita, Osaka 565-0871, Japan \\ E-mail:sukawa@thd.mech.eng.osaka-u.ac.jp
}

\begin{abstract}
In the present investigation, detailed observation on the pressure drop characteristics affected by the wall wettability in a curved mini-channel were carried out. The test channel is the serpentine channel with six straight sections and curved sections. The test channel has a square cross section that has $2.0 \mathrm{~mm}$ on each side, and the radius of curvature was $1.0 \mathrm{~mm}$. The test channel was made of the transparent acrylic resin for flow visualization. In order to investigate the influence of the wall wettability, water-repellent and attracting treatments were applied to the acrylic channel, respectively. In this investigation, in relation to the analysis method of the pressure drop, we evaluated the time averaged pressure drop and the pressure fluctuation, respectively. In the case of the water-repellent and attracting channel, the time averaged pressure drop was lower than the normal channel. In addition, in the straight section without the water repellent, the fluctuation of the pressure drop was considered to be correlated only by the Lockhart-Martinelli's parameter. On the other hand, in the cases the effects of channel curvature and water repellent are outstanding, the fluctuation of the pressured drop was widely scattered and hard to be correlated by the Lockhart-Martinelli's parameter.
\end{abstract}

Key words: Two-Phase Flow, Curved Mini-Channel, Wettability, Pressure Drop

\section{Introduction}

As machine become smaller, gas/liquid two-phase flow in a curved mini-channel is encountered in many applications such as nuclear engineering, aerospace engineering, mechanical engineering and environmental engineering. In particular, in nuclear engineering, two-phase flow heat transfer and CHF in mini-channel plays predominant role in cooling the melted core in the pressure vessel. In order to improve the performance of such small-scale devices, the information of gas/liquid two-phase flow in the small channel and its mechanism in the flow passage is required. Compared with the two-phase flow in ordinary diameter channel, the fundamental knowledge concerned with the small scale channels such as flow patterns, pressure drop, which are critical for the engineering design and practical performance, however, are not established enough. The most drastic difference between the mini-channel and ordinary diameter channel is the contribution of the surface tension force. The flow channels with hydraulic diameters of the order of, or smaller than the Laplace constant (Triplett et al, (1999)), gives out a clear criterion to identify whether the surface tension force surpasses the gravitational force. Under atmospheric pressure, this 
length scale equals to $6.3 \mathrm{~mm}$ for boiling water, while it is around $2.7 \mathrm{~mm}$ for air-water system at a room temperature. Two-phase flows with phase transition in narrow channel are also encountered in many applications. In recent years, Revellin and Thome (2007) investigated two-phase pressure drops over a wide range of experimental test conditions in two sizes of microchannels (sight glass tubes 0.509 and $0.790 \mathrm{~mm}$ ) for two refrigerants (R-134a and R-245fa). Furthermore, an actual two-phase flow system is rather a gas-liquid-solid system that is characterized by the surface tension between the gas and liquid phases and also by the contact angle between the liquid-gas interface and the channel wall. So, it is necessary to investigate the wall wettability effect due to the resin wetting characteristics and processing to form channels. Barajas and Panton (1993) investigated the effect of the contact angle upon the flow patterns with a $1.6 \mathrm{~mm}$ horizontal tube. The adiabatic flow of the air-water mixtures was tested over a wide range of the flow rates. In recent years, Lee and Lee (2007) investigated the effect of interfacial tensions on transition of two-phase flow pattern in mini-channels. Furthermore, the curvature effect in a curved mini-channel is necessary to investigate in order to improve the performance of the mini-devices, because the serpentine channel with many curved sections is ordinary used in many devices. In recent years, the radii of the curvature of the mini-scale devices becomes much smaller, it is considered that the curvature is becoming large. In such as polymer electrolyte fuel cells, there are a lot of hairpin curved sections to enlarge the chemical reaction area and to save volume of devices. So, it is necessary to have a detailed understanding of the flow characteristics on gas/liquid two-phase flow in the case that the curvature is comparatively large. In addition, in order to design the mini-scale devices that have a lot of mini-channels branched from a main channel, it is indispensable to investigate not only pressure drop but also its fluctuation, because of the adequate flow distribution to the branched channels. Such as polymer electrolyte fuel cells, there are mini-channels with the water-repellent.

The objectives of this study are to reveal the pressure drop characteristics by the wall wettability of curved mini-channel in the case which the curvature is comparatively large. In order to meet the condition which does not occur phase transition such as polymer electrolyte fuel cells, the experiment are performed under the room temperature and atmospheric pressure. The detailed observation for flow pattern by using the high speed video camera system and the precise measurements for pressure drop and its fluctuation are carried out to investigate the effects of the wall wettability on the gas/liquid two-phase flow in the small-scale channel.

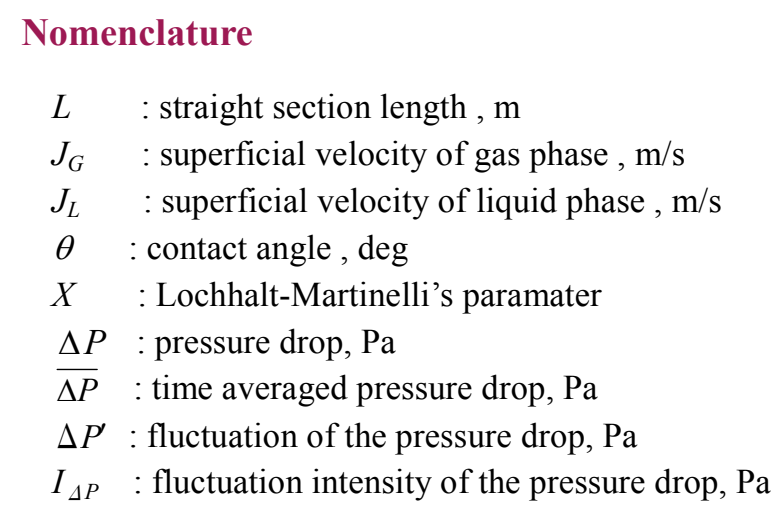

\section{Subscripts}

\footnotetext{
$G \quad$ : gas phase

$L \quad$ : liquid phase
} 


\section{Experiments}

\subsection{Experimental apparatus}

Figure 1 shows the schematic of the test section. The test section is the serpentine channel with six straight sections and curved sections. It is made of transparent acrylic block for flow visualization. The test channel has a square cross section that has $2.0 \mathrm{~mm}$ on each side. The length of the straight section is $94 \mathrm{~mm}$, and the interval between channels is $6 \mathrm{~mm}$. The gas/liquid mixing section is installed in the entrance of the test section. Figure 2 shows the detailed figure of the curved sections. The radius of the curvature is $1.0 \mathrm{~mm}$. The round marks (O) of Fig. 1 and Fig. 2 indicate the position of the pressure taps. Figure 3 shows the schematic of the set up of the experimental apparatus. The working fluids were air and distilled water for gas and liquid phase, respectively. The air was supplied by a compressor and the distilled water was circulated in the loop by a gear pump. The flow rate of the air and the distilled water was adjusted to the prescribed value by using the flow meter. The mixing section is placed at the entrance of the test section and is equipped with the two holes which are opposing. The radii of the two holes are $2.0 \mathrm{~mm}$ and $0.8 \mathrm{~mm}$, respectively. The air is supplied by the hole whose radius is $2.0 \mathrm{~mm}$, and water is supplied by the hole whose radius is $0.8 \mathrm{~mm}$.

\subsection{Experimental condition}

In order to observe the flow pattern of almost all the ranges from bubbly flow to rivulet flow, the superficial velocities of the gas and liquid were widely ranged from $0.083 \mathrm{~m} / \mathrm{s}$ to $20.8 \mathrm{~m} / \mathrm{s}$ for the gas-phase, and $0.042 \mathrm{~m} / \mathrm{s}$ to $1.67 \mathrm{~m} / \mathrm{s}$ for the liquid-phase, respectively. The experiment was performed under the room temperature and atmospheric pressure.

\subsection{Experimental procedures}

\subsubsection{Flow pattern}

The flow behavior of the two-phase flow was observed by using a digital high speed video camera. The camera was focused at around the curved section. The background illumination was provided by the metal halide lamp. In order to observe correctly the gas/liquid interface which moves comparatively at high speed, the shutter speed was set to be $1 / 8000 \mathrm{sec}$ and the frame speed was set to $4000 \mathrm{frame} / \mathrm{sec}$. It is enough to capture clear images of the gas/liquid two-phase flow in small channels. These images were then reproduced in slow motion for detailed observation.

\subsubsection{Pressure drop}

The pressure drop was measured with a precise differential pressure transducer (Valydyne, DP15-32). This differential pressure transducer has high time resolution and can measure correctly not only the time averaged value but also the fluctuation. The output of the pressure drop was sampled at the frequency of $1 \mathrm{kHz}$ for 20 seconds and recorded directly on a personal computer by using the A-D converter.

\subsubsection{Surface treatment}

To examine the influence of the wall wettability on the pressure drop characteristics, water-repellent and attracting treatment are applied to the acrylic channels wall, respectively. In the case of the water-repellent treatment, fluoride coating (Flurotechnology, FG-5010) was applied to the acrylic channels wall. In the case of the water-attracting treatment, photocatalyst coating was applied to the acrylic channels wall. Figure 4 shows the comparison of the static contact angles of each channel wall. Each of the static contact angles were measured on a flat sample of each channel resin. The contact angle without 
surface treatment was $64 \mathrm{deg}$. On the other hand, the contact angle with the water-repellent treatment was $104 \mathrm{deg}$. In addition, the contact angle with the water-attracting treatment was under $5 \mathrm{deg}$.

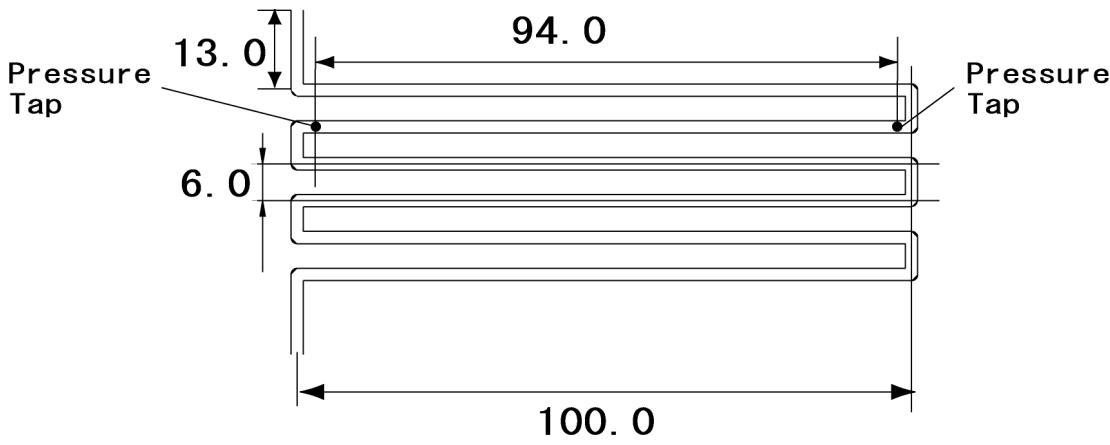

Fig. 1 Schematic of the test section

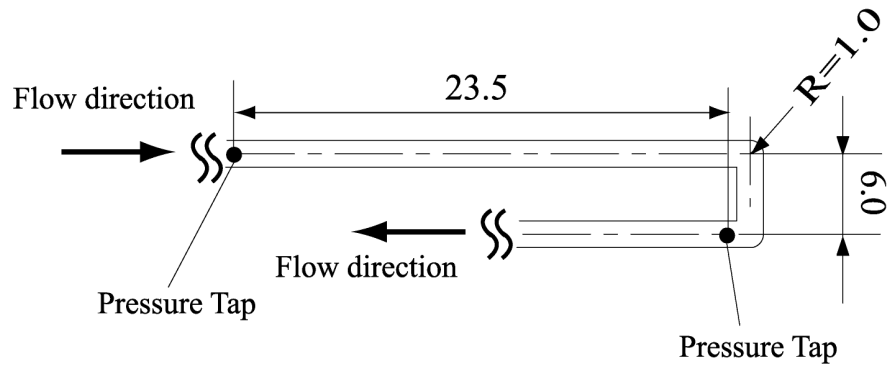

Fig. 2 Detailed figure of the curved section

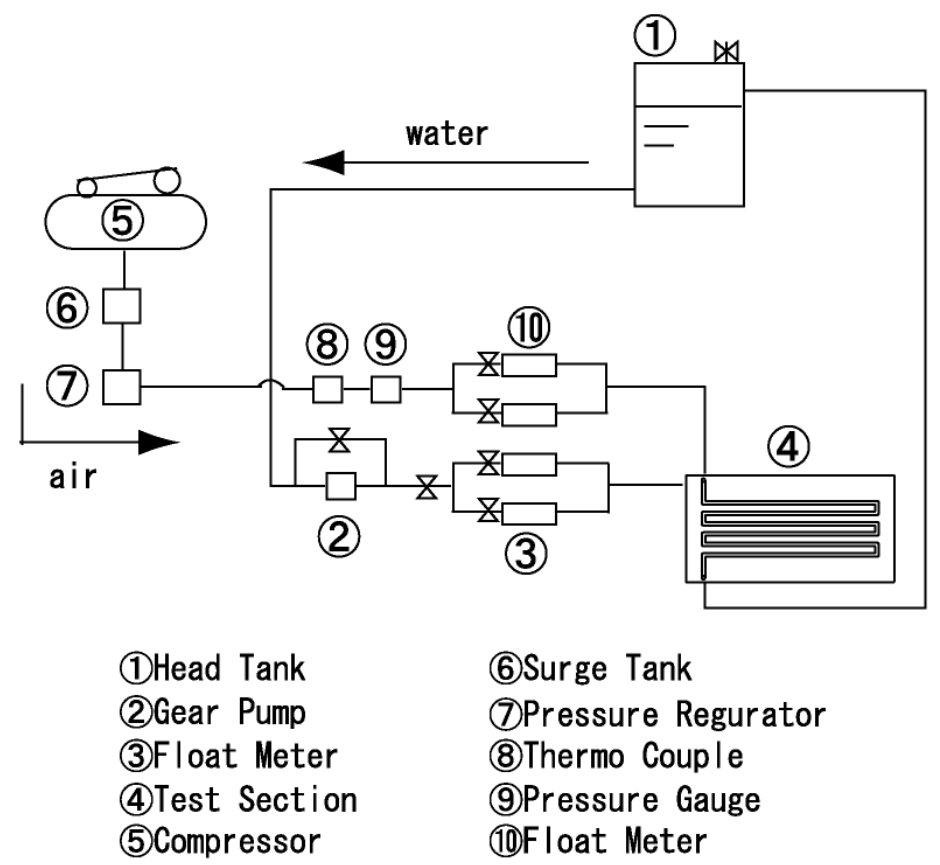

Fig. 3 Schematic of the curved section 


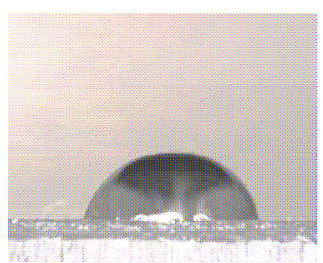

Without the surface treatment $\left(\theta=64^{\circ}\right)$ (Normal channel)

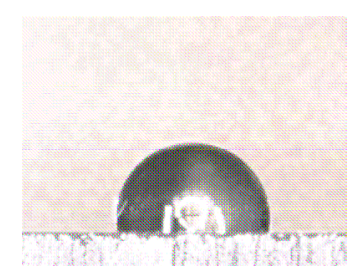

With the water-repellent treatment $\left(\theta=104^{\circ}\right)$ (Water-repellent channel)

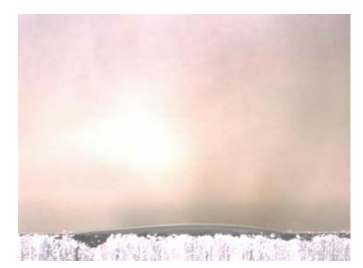

With the water-attracting treatment $\left(\theta<5^{\circ}\right)$

(Water-attracting channel)

Fig. 4 The comparison of the static contact angles

\section{Results and Discussion}

\subsection{Flow patterns}

Figure 5 shows the typical flow patterns observed in this study. The flow pattern, which we can see in this study were classified into the following six flow patterns.

The bubbly flow [Fig. 5(a) ] was characterized by distinct and distorted bubbles, generally smaller than the channel diameter. When the flow passes the curved section, the liquid phase flows outside and air bubbles flows inside due to the centrifugal force. The bubbly- slug flow [Fig. 5(b) ] was considered as the flow that the slug flow and bubbly flow were mixed. When the flow passes the curved section, the liquid phase distributes at the outside of the channel and gas is near the inside of the channel. The slug flow [Fig. 5(c) ] was considered as the flow that liquid slug and gas slug flowed by turns and the length is the almost same. The slug-annular flow [Fig. 5(d) ] was considered as the flow that the quite long slug and short liquid slug flowed by turns. The annular flow [Fig. 5(e) ] was considered as the flow that the liquid film formed along the channel wall and the gas flows through the center. The rivulet flow [Fig. 5(f) ] was considered that liquid film adhered the channel wall and uniform liquid film was not observed at all.

The stratified flow could not be observed in this study. In the mini-channel with the water-repellent, the rivulet flow was observed due to the condition of the stratified intermittent transition. Since the mini-channel with the water-repellent had lower surface tension at the interface between the solid and the liquid phases, it was considered that the uniform liquid film was difficult to form on the surface of the wall. For the above reason, it was considered that the mini-channel with the water-repellent was much easier to be formed rivulet flow than the without the water repellent.

Figure. 6 shows the specific phenomena observed in the curved section. When the slug-annular flow passed through the curved section, we could see the staying small bubbles near the entrance and exit in the curved section. Furthermore, after passing the curved section, it returned the original slug-annular flow. In the curved section, it was considered that the stagnation region is formed in the inside of the channel due to the sudden curved section. In addition, the liquid flowed outside, air bubbles flowed inside due to the centrifugal force. Because of the strong shear force around the curved section, air bubbles were separated from the gas slug and stayed in the inside of the channel. Regardless of the surface treatment, we could see this specific phenomenon. 


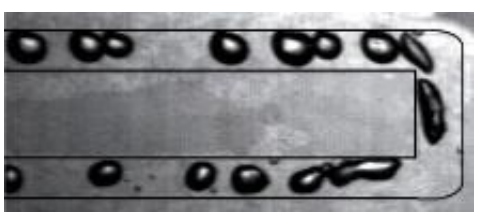

(a) $J_{G}=0.139[\mathrm{~m} / \mathrm{s}]$ $J_{L}=0.833[\mathrm{~m} / \mathrm{s}]$

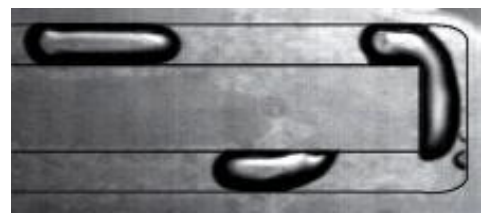

(c) $J_{G}=0.125[\mathrm{~m} / \mathrm{s}]$

$J_{L}=0.292[\mathrm{~m} / \mathrm{s}]$

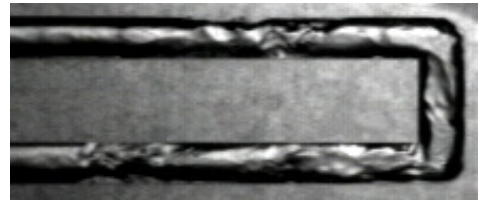

(e) $J_{G}=15.9[\mathrm{~m} / \mathrm{s}]$ $J_{L}=0.417[\mathrm{~m} / \mathrm{s}]$

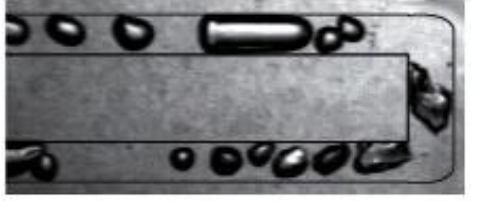

(b) $J_{G}=0.274[\mathrm{~m} / \mathrm{s}]$

$J_{L}=0.750[\mathrm{~m} / \mathrm{s}]$

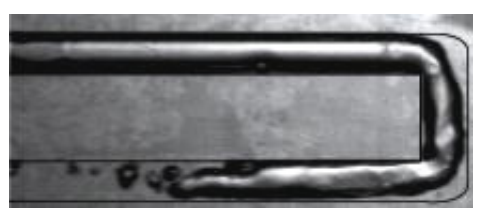

(d) $J_{G}=1.35[\mathrm{~m} / \mathrm{s}]$

$J_{L}=0.417[\mathrm{~m} / \mathrm{s}]$

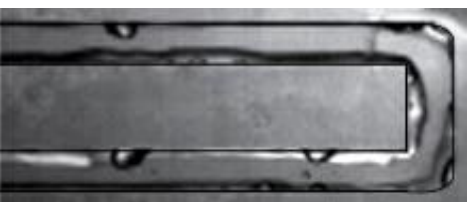

(f) $J_{G}=13.1[\mathrm{~m} / \mathrm{s}]$

$J_{L}=0.042[\mathrm{~m} / \mathrm{s}]$

Fig. 5 Typical examples of the flow

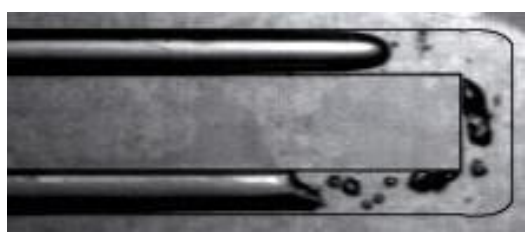

(e) $J_{G}=1.35[\mathrm{~m} / \mathrm{s}]$

$J_{L}=0.042[\mathrm{~m} / \mathrm{s}]$

Fig. 6 Specific phenomenon in the curved section

\subsection{Flow patterns}

We summarized the flow pattern maps shown in Figs. 7.1-7.3. In these figures, the abscissa is superficial velocity of gas-phase and the ordinate is superficial velocity of liquid phase. Figure 7.1 shows the flow pattern map of the normal channel. In the case of the normal channel, when the superficial velocity of gas phase increases at low superficial velocity of liquid phase, flow pattern changes to slug-annular flow from slug flow, eventually changes to annular flow. On the other hand, when the superficial velocity of gas phase increases at high superficial velocity of liquid phase, flow pattern changes to bubbly-slug flow from bubbly flow and changes to slug-annular flow in turn, eventually changes to annular flow.

Figure 7.2 shows the flow pattern map of the water-repellent channel. In the case of the water-repellent channel, when the superficial velocity of gas phase increases at low superficial velocity of liquid, flow pattern changes to slug-annular flow from slug flow and changes to annular flow in turn, eventually changes to rivulet flow. On the other hand, when the superficial velocity of gas phase increases at high superficial velocity of liquid phase, 
flow pattern changes to annular flow. So, by comparing the normal channel and the water-repellent channel, it is especially noted that the boundary of slug-annular and annular is changed by the effect of the wall wettabiliy. Since the water-repellent channel had lower surface tension at the interface between solid and liquid phase, it is considered that the liquid is moved by shear force much easier than without the surface treatment.

Figure 7.3 shows the flow pattern map of the water-attracting channel. In the case of the water-attracting channel, when the superficial velocity of gas phase increases at low superficial velocity of liquid phase, flow pattern changes to slug-annular flow from slug flow, eventually changes to annular flow. On the other hand, when the superficial velocity of liquid phase increases at high superficial velocity of liquid phase, flow pattern changes to bubbly-slug flow from bubbly flow and changes to slug-annular flow in turn, eventually changes to annular flow. So, by comparing the normal channel and the water-attracting

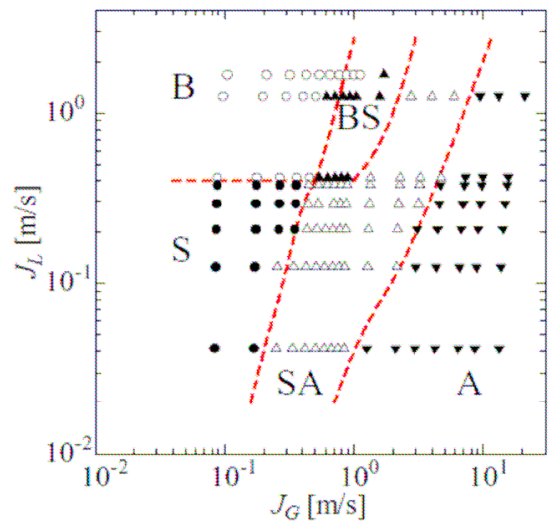

Fig. 7.1 Flow pattern map of the normal channel

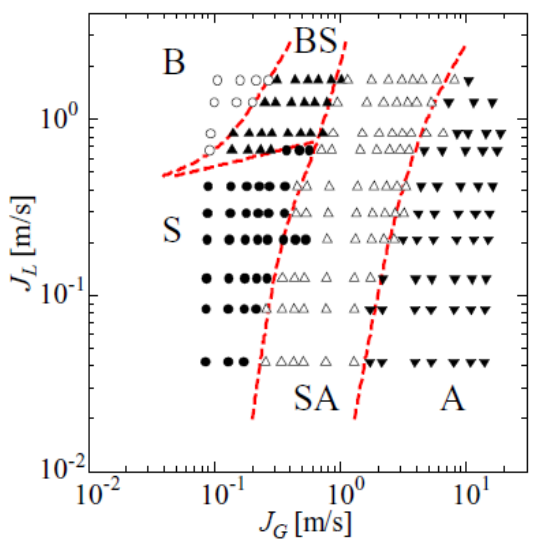

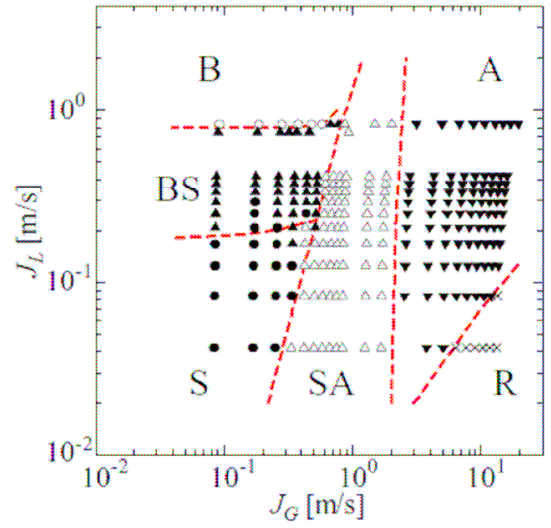

Fig. 7.2 Flow pattern map of the water-repellent channel

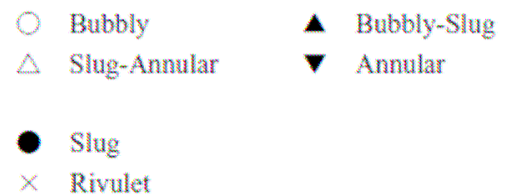

Fig. 7.3 Flow pattern map of the water-attracting channel

channel, it is especially noted that the boundary of bubbly and bubbly-slug is changed by the effect of wall wettability. When the bubbles are formed in the channel, it is considered that the solid-gas-liquid interface is need to be formed in the mixing section. So, in the case of the water-repellent channel, it is considered that solid-gas-liquid interface forms easily in the mixing section. On the other hand, in the case of the water-attracting channel, it is considered that solid-gas-liquid interface is hard to be formed in the mixing section.

\subsection{Pressure drop characteristics}




\subsubsection{Time-averaged pressure drop}

Figure 8 shows the comparison of the time averaged pressure drop of each channel. The ordinate of this figure shows $\overline{\Delta P} / L$ which is the time-averaged pressure drop normalized by the length of the straight section. The abscissa shows the superficial velocity of gas phase.

In the case of the normal channel, $\overline{\Delta P} / L$ increases with increasing of the superficial velocity of gas phase, and had maximum value when a flow pattern changed to slug-annular flow from slug flow. After taking maximum value, $\overline{\Delta P} / L$ once decreases with increasing superficial velocity of gas phase, and increases again. In the case of the water-repellent channel, $\overline{\Delta P} / L$ increases monotonously with increasing superficial velocity of gas phase, and had maximum value where the flow pattern changed to slug-annular flow from bubbly-slug flow. However, in the case of the water-repellent channel, it is noted that maximum value of the pressure drop is quite lower than the normal channel. In the case of the water attracting-channel, it also had a maximum value when the flow pattern changed to slug-annular flow from slug flow. Although maximum value of the pressure drop is lower than the normal channel, it is higher than that of the water-repellent channel. As compared with the corresponding flow pattern, it is noted that the time-averaged pressure drop takes maximum value when the liquid slug is formed in the channel. As mentioned in the Fig. 8, the time-averaged pressure drop was also changed by the wall wettability, which can affect the flow pattern transition. In the normal channel, the peak value takes at the slug flow regime. In the water-repellent channel, the peak of the pressure drop takes at the bubbly-slug flow. The difference of the corresponding flow pattern might be the reason why the peak value is lower in water-repellent channel compared with the normal channel. In the water-attracting channel, the maximum value is also lower than the normal channel despite the flow pattern is the same, slug flow. Following might be the reason why the peak value of the pressure drop decreases in water-attracting channel compared with the normal channel. It is noted that the liquid film formation on the channel wall is changed by the wall wettability. In the water-attracting channel, the whole channel wall is always wet, that is, the micro-scale liquid film remains even though in the slug flow regime. On the other hand, in the normal channel, the liquid film cannot keep at the gas-slug part and the wall is dried. The contact angle of the liquid meniscus might be changed by the wall wettability. So, it is considered that the liquid slug moves easily and the pressure drop peak decreases in water-attracting channel as compared with the normal channel.

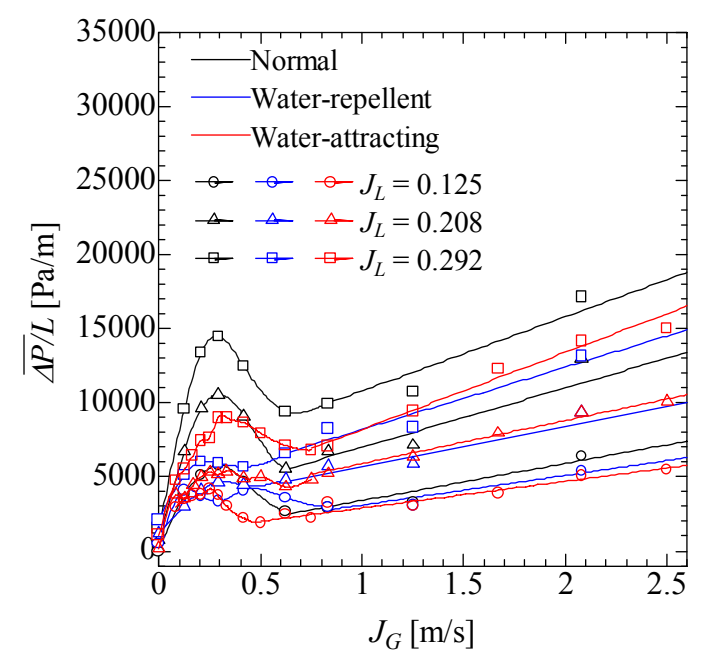

Fig. 8 The comparison of the time averaged pressure drop of each channel

\subsubsection{Pressure fluctuation intensity}


In this study, concerning the fluctuation of the pressure drop for gas/liquid two-phase flow, we used the pressure fluctuation intensity, $I_{\Delta P}$. The pressure fluctuation intensity, $I_{\Delta P}$, was defined by the flowing equations.

$$
\begin{aligned}
& I_{\Delta P}=\frac{\sqrt{\left(\Delta P^{\prime}\right)^{2}}}{\overline{\Delta P}} \\
& \Delta P=\overline{\Delta P}+\Delta P^{\prime}
\end{aligned}
$$

$I_{\triangle P}$, was defined as the standard deviation of the pressure fluctuation component normalized by the time-averaged pressure drop. Figures 9-11 show the pressure fluctuation intensity of the straight section. On the other hand, Figs. 12-14 show the pressure fluctuation intensity of the curved section. In these figures, the six kinds of plotted symbols indicate the flow patterns, respectively. The experimental results were summarized by the Lockhart-Martinelli's parameter $X$. From these figure, regardless of the wall wettability, it was noted that the pressure fluctuation intensity, $I_{\Delta P}$, has maximum value for around $X=2$. The flow pattern of this condition corresponds to the slug-annular flow. This means that the pressure fluctuation intensity reaches a peak value in the slug-annular flow regime. In slug-annular flow regime, the liquid slug can be formed easily and passes intermittently through the test section. That causes the pressure fluctuation becoming large.

From the Fig. 9, 11, in the straight section of the normal and water-attracting channel, it is also noted that the pressure fluctuation, $I_{\Delta P}$, does not so much scattered and could be correlated by the Lockhart-Martinelli's parameter. This means that the pressure fluctuation is dominant only by the Lockhart-Martinelli's parameter, which represents consequentially the flow ratio of gas and liquid phases. From the Fig. 10, however, in the straight section of the water-repellent channel, it could be pointed out that the data is widely scattered as compared with the case of the normal and water-attracting channel. This means that the pressure fluctuation is hard to be correlated only by the Lockhart-Martinelli's parameter due

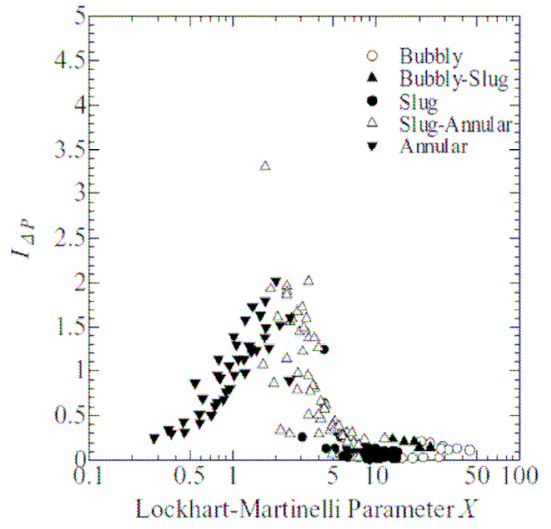

Fig. 9 Pressure fluctuation intensity of the straight section with the normal channel

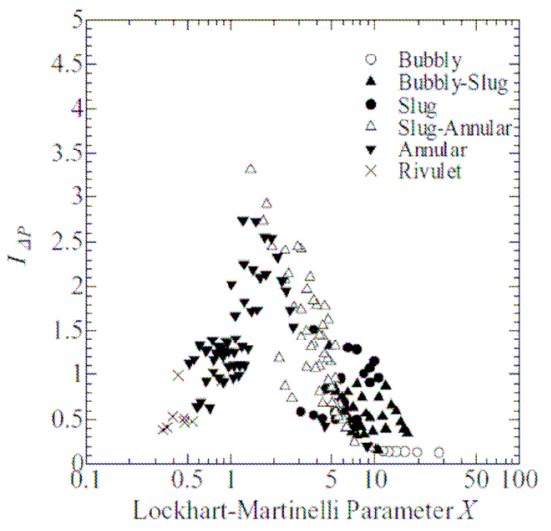

Fig. 10 Pressure fluctuation intensity of the straight section with the water-repellent channel 


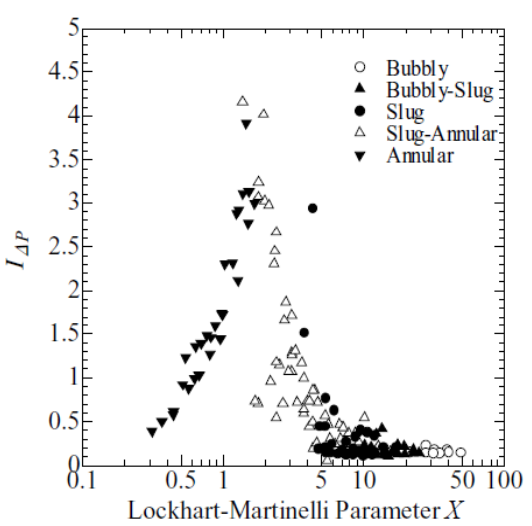

Fig. 11 Pressure fluctuation intensity of the straight section with the water-attracting channel

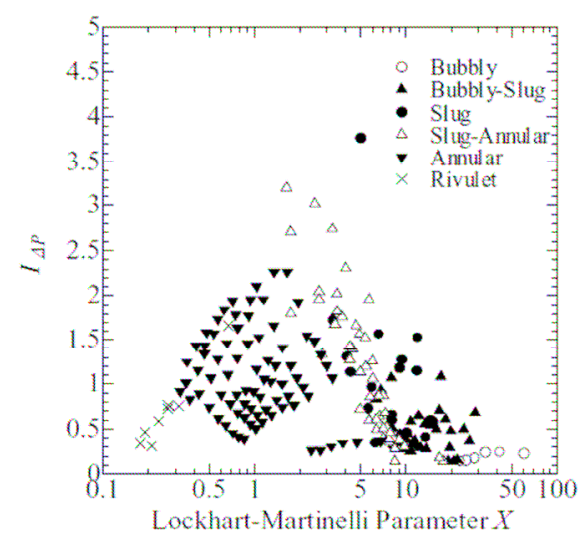

Fig. 13 Pressure fluctuation intensity of the curved section with the water-repellent channel

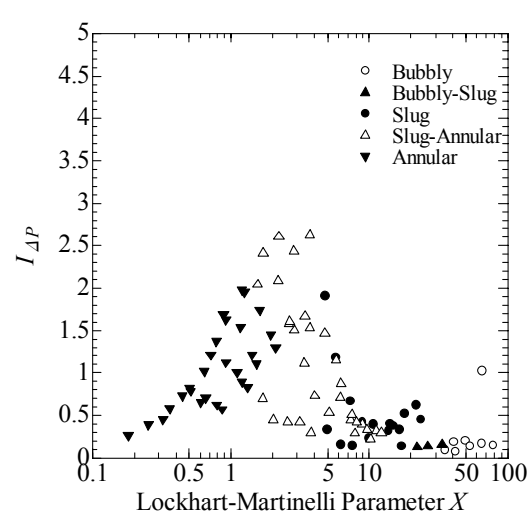

Fig. 12 Pressure fluctuation intensity of the curved section with the normal channel

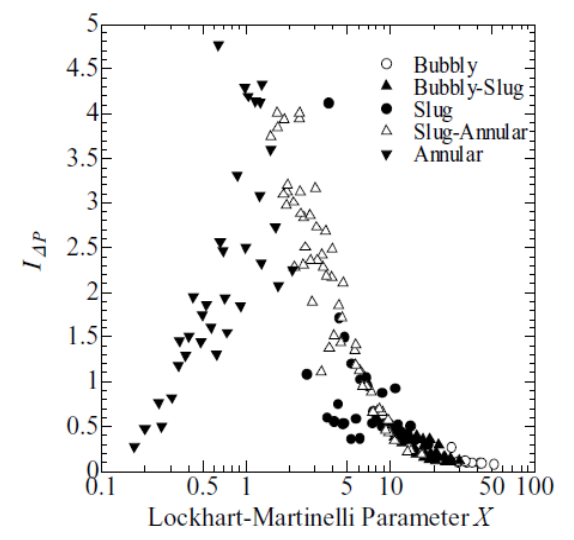

Fig. 14 Pressure fluctuation intensity of the curved section with the water-attracting channel

to the effect of the wall wettability. From the Fig. 11, in the water-attracting channel, it is noted that the pressure fluctuation becomes larger than the other channels when the pressure fluctuation takes maximum value for around $X=2$. In addition, from the Figs. 7.1-7.3, in the water-attracting channel, the regime of the slug flow and slug-annular flow is wider than the other channels. So, in the straight section with the water-attracting channel, it is considered that the liquid slug forms easier than the other channels and the pressure fluctuation becomes large.

From the Fig. 12-13, in the curved section with the normal and water-repellent channel, the data is widely scattered as compared with the straight section. This indicates that the pressure fluctuation can no longer be correlated by Lockhart-Martinelli's parameter. In addition, the pressure fluctuation becomes generally larger than the straight section. However, in the case of the normal and water-repellent channel, there are cases where the pressure fluctuation becomes lower than the straight section. This tendency is especially significant in the annular flow regime with the water-repellent channel. In order to clear in detail what is happening in the curved section with the water-repellent channel, we choose typical two cases of the annular flow where the Lockhart-Martinelli's parameter is nearly the same value. First one is the case of annular flow where the pressure fluctuation intensity is large. Second one is the case of annular flow where the pressure fluctuation intensity is small. Figure 15 shows the comparison of the annular flow in the water-repellent channel. Figure 15.1 shows the pressure fluctuation intensity of the curved section with the 
water-repellent channel. Figure 15.2 shows the flow pattern map of the water-repellent channel. In these figures, (a) indicates the annular flow where the pressure fluctuation is large, (b) indicates the annular flow where the pressure fluctuation is small. The round mark

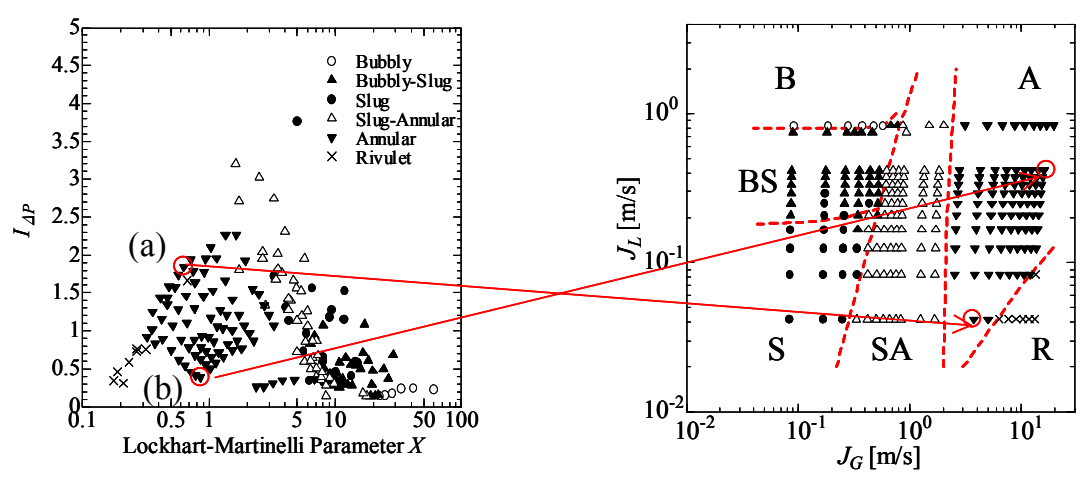

Fig. 15.1

Fig. 15.2
(a) $J_{G}=3.81[\mathrm{~m} / \mathrm{s}] \quad J_{L}=0.083[\mathrm{~m} / \mathrm{s}]$
(b) $J_{G}=15.9[\mathrm{~m} / \mathrm{s}] \quad J_{L}=0.417[\mathrm{~m} / \mathrm{s}]$

Fig. 15 The comparison of the annular flow with the water-repellent channel

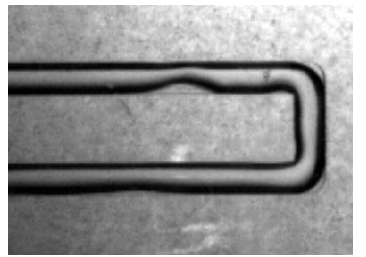

Fig. 16 Annular flow where the pressure fluctuation intensity is large (Water-repellent channel) $\left(J_{G}=3.81[\mathrm{~m} / \mathrm{s}] J_{L}=0.083[\mathrm{~m} / \mathrm{s}]\right)$

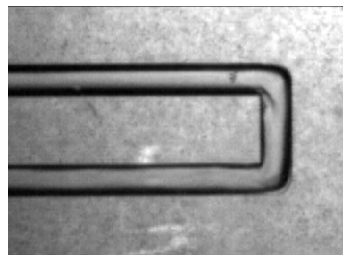

Fig. 17 Annular flow where the pressure fluctuation intensity is small (Water-repellent channel) $\left(J_{G}=15.9[\mathrm{~m} / \mathrm{s}] J_{L}=0.417[\mathrm{~m} / \mathrm{s}]\right)$

$(\bigcirc)$ in Fig. 15.1 corresponds to the round mark $(\bigcirc)$ in Fig. 15.2. This shows that (a) corresponds to the case with relative low mass flux, and (b) is the case with high flux, respectively. Figure 16 and Figure 17 show the direct photograph of the annular flow indicated by (a) and (b) in Fig. 15.1, respectively. As shown in Fig. 16, the liquid film flowing on the wall has wavy interface. Large waves are formed and nearly choke up the channel. This large wave occurs intermittently in this case. On the other hand, in the case with high flux shown in Fig. 17, the gas liquid interface becomes smoother as compared with Fig. 16. In general, in the water-repellent channel, the gas-liquid interface tends to be unstable due to the water-repellent characteristics of the wall. However, as superficial velocity is large, it is considered that the phase separation of the two phases occurs easily due to the strong shear acting on the gas-liquid interface and the centrifugal force in the curved section. This is the main reason why the pressure fluctuation in high mass flux case in the curved section becomes smaller.

From the Fig. 14, in the case of the curved section with the water-attracting channel, the data is not widely scattered as compared with the straight section. This means the pressure fluctuation can be generally correlated by Lockhart-Martinelli's parameter. However, the pressure fluctuation becomes generally larger than the other channels. Figure 18 shows the photograph of the annular flow in the water-attracting channel. The superficial velocity of this case is nearly the same with the case in water-repellent channel shown in Fig. 17. As shown in Fig. 18, the gas-liquid interface becomes wavy despite the superficial velocity is 
relatively large. In the water-attracting channel, even though in the curved section affected by the centrifugal force, the liquid film adhered well to the inside wall. This indicates that the liquid film tends to stay and grow to the large wave at the inside of the curved section. So, in the water-attracting channel, the pressure fluctuation becomes larger than the other channels.

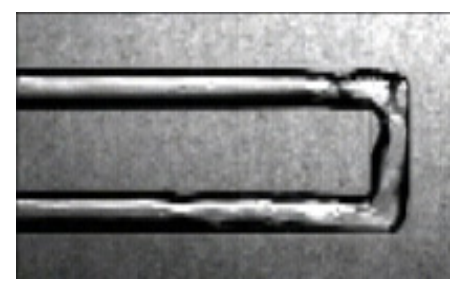

Fig. 18 Annular flow where the superficial velocity corresponds to the Fig. 17.

(Water-attracting channel)

$\left(J_{G}=15.9[\mathrm{~m} / \mathrm{s}] J_{L}=0.417[\mathrm{~m} / \mathrm{s}]\right)$

\section{Conclusions}

We experimentally examined the pressure drop characteristics affected by the wall wettability in a curved mini-channel. Two-phase flow patterns, time-averaged pressure drop and pressure fluctuation were measured and analyzed. Based on the results, the following conclusions can be extracted:

(1) Since surface tension at the interface between the solid and liquid phase is changed by the wall wettability, the flow pattern and transition boundaries are strongly influenced by the channel wall condition.

(2) As radius of the curvature becomes smaller, shear forces around the curved section becomes stronger due to the centrifugal force and the occurrence of the stagnation region.

(3) Flow pattern transition and liquid film formation are changed by the wall wettability. So, the time-averaged pressure drop is changed by the channel wall condition.

(4) The pressure fluctuation intensity has a maximum value around Lockhart-Martinelli's parameter $X=2$. The flow pattern of this condition corresponds to the slug-annular flow. In the case of channel wall that liquid slug forms easily, pressure fluctuation becomes large.

(5) In the straight section except as water-repellent channel, the fluctuation of the pressure drop could be correlated only by the Lockhart-Martinelli's parameter, which is consistent with previous works.

(6) On the other hand, the fluctuation of the pressure drop in the curved section was hard to be correlated by the Lockhart-Martinelli's parameter due to the effects of centrifugal force. The effect of wall wettability can also affect on the pressure drop fluctuation characteristics. 


\section{References}

(1) Suo, M. and Griffith, P., Two-phase flow in capillary tubes, J. Basic Engineering, Vol.86 (1964), pp576-582.

(2) Barnea, D., Shoham, O. and Taitel, Y., Flow pattern in horizontal and vertical two-phase flow in small diameter pipes, The Can. J. Chemical. Engineering, Vol.61 (1983), pp617-620.

(3) Fukano, T., Kariyasaki, A. and Kagawa, M., Flow patterns and pressure drop in isothermal gas-liquid concurrent flow in a capillary tube, Transactions of the Japan Society of Mechanical Engineers, Series B, Vol.56, No.528 (1990), pp.2318-2326.

(4) Fukano, T. and Kariyasaki, A., Characteristics of gas-liquid two-phase flow in a capillary tube, Nuclear Engineering and Design, Vol.141 (1993), pp.59-68.

(5) Tripllet, K.A., Ghiaasiann, S.M., Adbel-Khalik, S.I. and Sadowski, D.L., Gas-liquid two-phase flow in microchannels Part I: Two-phase flow pattern, Int. J. Multiphase Flow, Vol.25 (1999), pp.377-394.

(6) Feng, Z.P. and Serizawa, A., A review of gas-liquid two-phase flow hydraulics in micro-channels, The proceedings of the US-Japan Seminar on Two-Phase Flow Dynamics, (2000).

(7) Barajas, A.M. and Panton, R.L., The effects of contact angle on two-phase flow in capillary tubes, Int. J. Multiphase Flow, Vol.19 (1993), pp.337-346.

(8) Kariyasaki, A., Fukano, T., Okada, A. and Kagawa, M., Isothermal air-water two-phase flow through a horizontal capillary return bend, Transactions of the Japan Society of Mechanical Engineers, Series B, Vol.62, No.593 (1996), pp.115-121.

(9) Mishima, K. and Hibiki, T., Some characteristics of air-water two-phase flow in small diameter vertical tubes, Int. J. Multiphase Flow, Vol.22 (1996), pp.703-712.

(10) Cubaud, T. and Ho, C., Transport of bubbles in square microchannels, Physics of Fluids, Vol.16, No.12 (2004), pp.4575-4585.

(11) Lee, C.Y. and Lee, S.Y., Influence of interfacial on transition of two-phase flow patter in mini-channels, Proc. 6th Int. Conf. Multiphase Flow(on CD-ROM), (2007).

(12) Revellin, R. and Thome, J., Adiabatic two-phase frictional pressure drops in microchannels, Experimental Thermal and Fluid Science, Vol.31 (2007), pp.673-685.

(13) Kawahara, A., Chung, P.M.-Y. and Kawaji, M., Investigation of two-phase flow pattern, void fraction and pressure drop in a maicrochannel, Int. J. Multiphase Flow, Vol.28 (2002), pp.1411-1435. 